Supporting Information for manuscript:

\title{
The importance of viscosity control for recyclable reinforced thermoplastic composites.
}

Gijs W. de Kort ${ }^{1, *}$, Sarah Saidi ${ }^{2,3}$, Daniel Hermida-Merino ${ }^{3}$, Nils Leoné ${ }^{1}$, Varun Srinivas ${ }^{1}$, Sanjay Rastogi ${ }^{1}$, Carolus H. R. M. Wilsens ${ }^{4, *}$.

${ }^{1}$ Aachen-Maastricht Institute of BioBased Materials (AMIBM), Maastricht University, P.O. Box 616, 6200MD, Maastricht, The Netherlands. 'LMOPS, EA 4423, Université de Lorraine, CentraleSupelec Metz, 2 rue Edouard Belin, Metz, F-57070, France. ${ }^{3}$ Netherlands Organisation for Scientific Research (NWO), DUBBLE@ESRF BP CS40220, 38043 Grenoble, France. 4 Sabic Technology \& Innovation, STC Geleen, Urmonderbaan 22, 6160AL, Geleen, The Netherlands

*Corresponding authors: gijs.dekort@maastrichtuniversity.nl, karel.wilsens@sabic.com

KEYWORDS: thermotropic polyester, blend, morphology, viscosity control orientation, relaxation

Supporting information: 6 pages, 7 Figures 


\section{Evaluation of the linear viscoelastic regime for the LCPs at different temperatures}

To ensure an accurate comparison between the viscoelastic behavior of the different LCPs, the viscosity of LCPP and CE-1:1 were measured as function of the applied strain at $150{ }^{\circ} \mathrm{C}$ and 190 ${ }^{\circ} \mathrm{C}$ (Figure S1). Both LCPs show a constant viscosity at strains below $10 \%$.

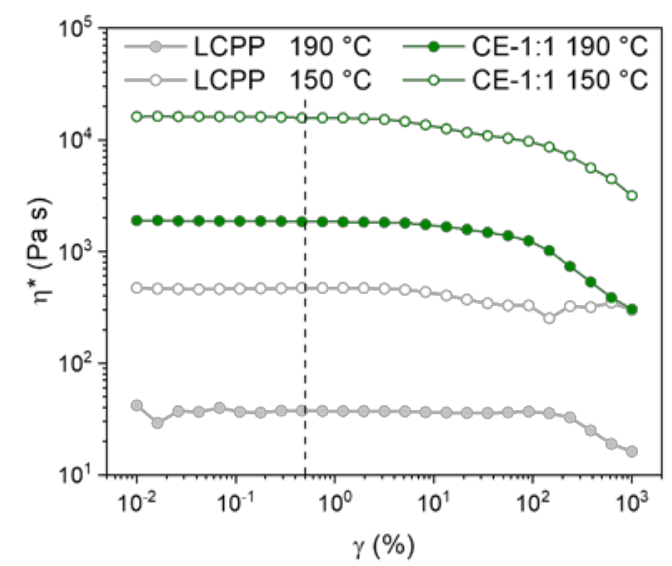

Figure S1. The complex viscosity of $\mathbf{L C P P}$ and $\mathbf{C E}-2: 1$ at $190{ }^{\circ} \mathrm{C}$ and $150^{\circ} \mathrm{C}$ as a function of oscillation strain $(\omega=$ rad s $\left.{ }^{-1}\right)$. The strain at which the frequency sweeps were measured is marked by the dotted line.

\section{Fibril morphology of injection molded bars containing $30 \mathrm{wt} \%$ of the respective LCPs}

As discussed in the main paper, all composites have a fine fibrillar morphology. The LCP particles, although clearly deformed, appeared short in the images taken from the microtomed slices with a thickness of $1 \mu \mathrm{m}$. To show that actual fibrils are formed in the injection molding process, POM images obtained from microtomed slices with a thickness of $2.5 \mu \mathrm{m}$ are displayed here. In all composites, fibrils with lengths over $100 \mu \mathrm{m}$ are observed, and the observed trends with respect to the fibril diameter and appearance are in agreement with those in the main paper.
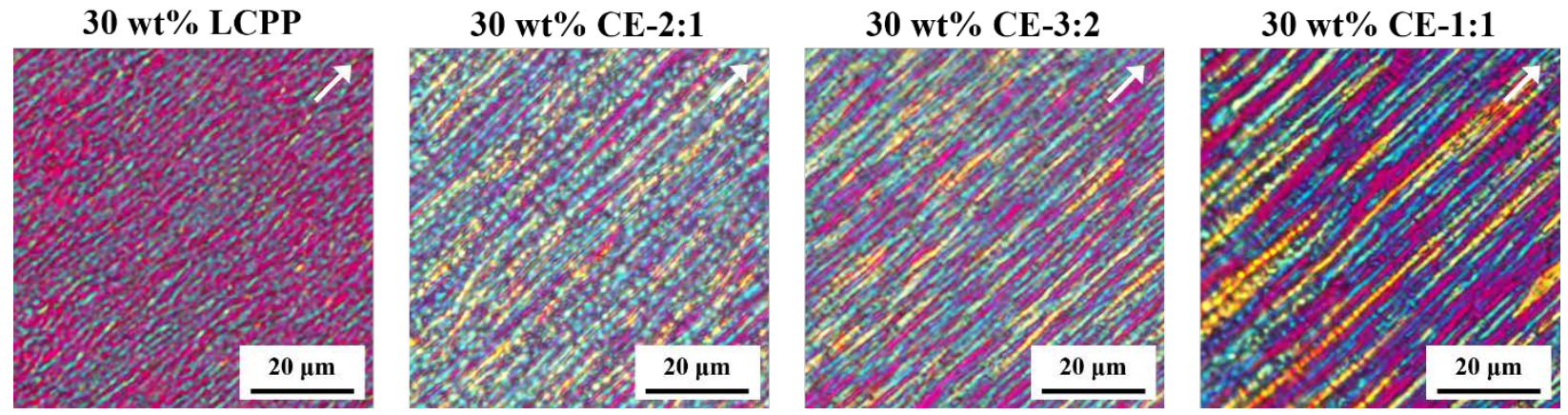

Figure S2. Morphology of injection molded composites containing the different LCPs. The composites were microtomed to slices with a thickness of $2.5 \mu \mathrm{m}$. The images represent the core-region of the sample. The white arrows mark the injection direction. 


\section{Layer morphology of injection molded bars containing $30 \mathrm{wt} \%$ of the respective LCPs}

Injection molded parts often display a layered morphology, caused by differences in the deformation rate and cooling rate the material experiences throughout the cross-section of the part. In the case of an LCP dispersed in a thermoplastic matrix, this is often expressed in differences in the LCP morphology. Five distinct layers are observed in all different LCP-PLLA blends, as shown in Figure S3: 1) a quench layer in direct contact with the wall, 2) a shear layer close to the wall, 3) a transition layer a bit further from the wall, 4) a second transition layer, 5) a core region at the center of the sample. In the quenched layer, the material is cooled extremely quickly by the mold wall, preventing the formation of oriented structure. This layer is in the order of $1 \mu \mathrm{m}$ for these samples. In the shear layer, where the material experiences high stresses but slightly lower cooling rates, the LCP is present in form of highly oriented platelets. The first transition layer characteristically has shorter, irregular fibrils, as the stresses are reduced at this position but the cooling rates remain relatively high. The second transition layer and core layer both have a fibrillar morphology, however the fibrils in the second transition layer are notably thicker and longer. In both these layers, the cooling rate is relatively low, due to thermal insulation by the other layers, but the higher stresses in the transition layer allows the formation of longer fibrils.

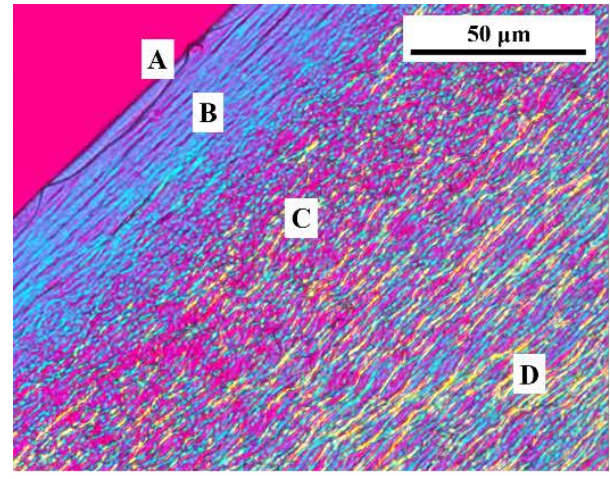

$30 \mathrm{wt} \%$ LCPP
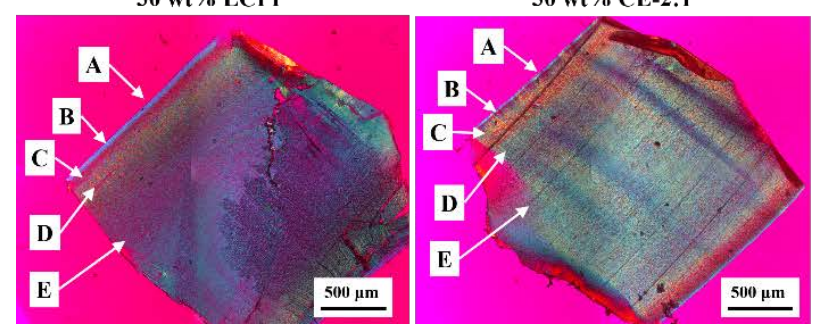

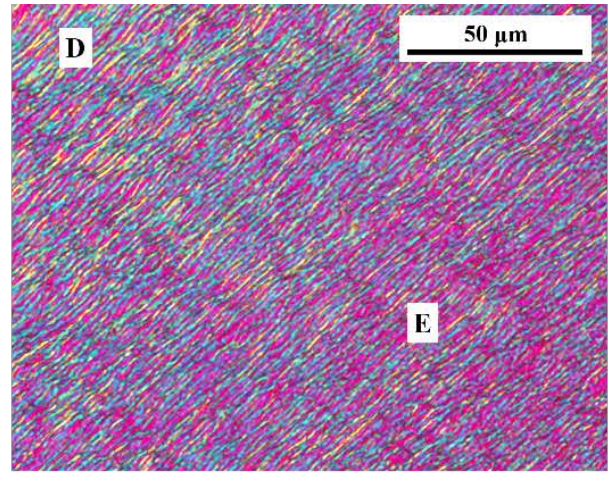

30 wt $\%$ CE-3:2

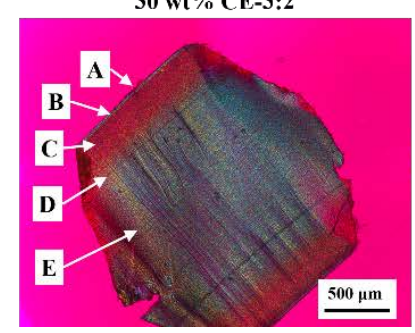

$$
\begin{aligned}
& \text { A }=\text { quench layer } \\
& B=\text { shear layer } \\
& C=\text { transition layer I } \\
& D=\text { transition layer II } \\
& E=\text { core }
\end{aligned}
$$

$30 w t \%$ CE-1:1

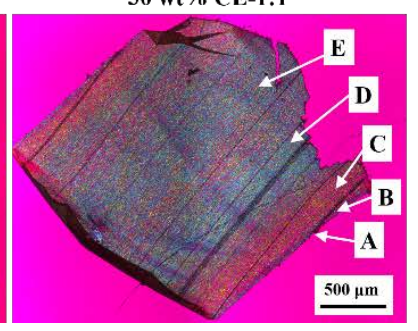

Figure S3. (top) polarized optical microscopy images of the morphology the $30 w t \%$ LCPP/PLLA composite. The different layers that are observed are labeled (A-E). Images were obtained from microtomed slices with a thickness of $2.5 \mu \mathrm{m}$. (bottom) Cross-sections of the LCP - PLLA composites. The different layers that are observed are labeled (A-E). Images were obtained from microtomed slices with a thickness of $2.5 \mu \mathrm{m}$. 
The effective viscosity ( $\eta_{\text {eff }}$ ) of a molten blend is affected by the presence of the different LCPs:

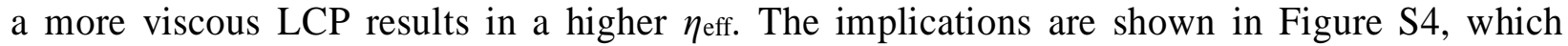
displays the trends in the thickness of each layer in the injection molded LCP/PLLA composites as function of the LCP viscosity.

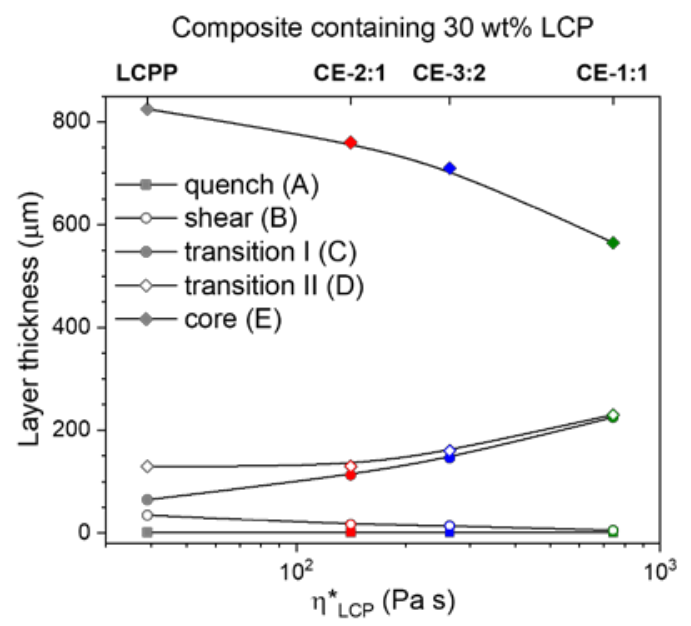

Figure S4. Trends in the thickness of the different layers as a function of the LCP viscosity as observed from the mircotomed cross-sections of the different LCP-PLLA composites. The viscosity of the different LCPs (measured in parallel plate rheology at $\omega=137 \mathrm{rad} \mathrm{s}^{-1}, \mathrm{~T}=190{ }^{\circ} \mathrm{C}$ ).

\section{Comparison of the viscoelastic behavior of semi-crystalline PLLA and amorphous PLA grades}

In this study, melt drawn tapes containing of $30 \mathrm{wt} \%$ of each of the respective LCPs are used investigate the relaxation behavior of the LCPs within a matrix phase. Two different PLLA grades are used as matrix: a semi-crystalline PLLA grade (low D-isomer content), and an amorphous PLA grade (higher D-isomer content). To allow a fair comparison, a similar morphology should be present in the tapes, and therefore the viscoelastic behavior of both PLLA grades needs to be comparable. This is confirmed via rheometry, as shown in Figure S5. The complex viscosity (Figure S5a) and the moduli (Figure S5b) of the different PLLAs are similar and show a similar temperature dependence. 
a

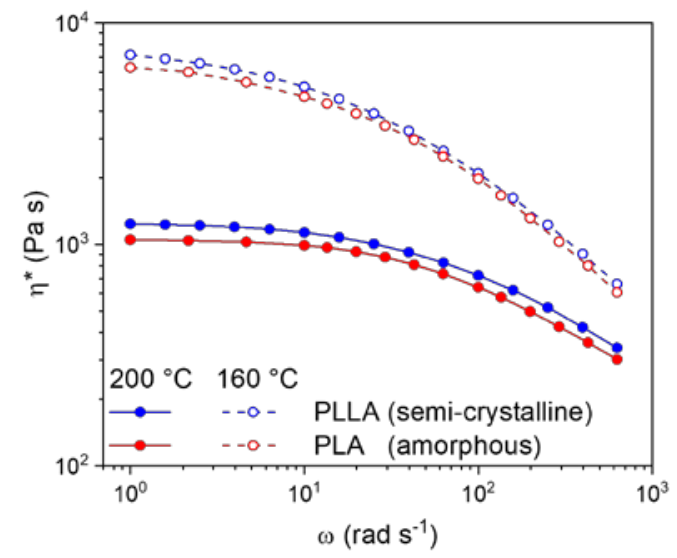

b

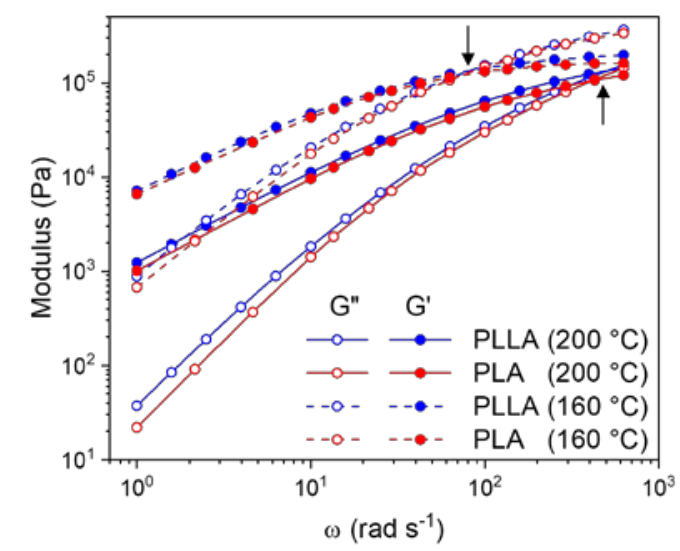

Figure S5. (a) Complex viscosity as a function of oscillation frequency of the semi-crystalline PLLA and amorphous PLA. (b) Viscous modulus and elastic modulus as a function of oscillation frequency of the semi-crystalline PLLA and amorphous PLA. The black arrows mark the respective crossover points.

\section{DSC traces of melt drawn tapes}

The thermal behavior of the melt drawn tapes is evaluated by differential scanning calorimetry (DSC) using a TA Instruments Q2000 DSC. The heating and cooling-rates of the sample were 10 ${ }^{\circ} \mathrm{C} \mathrm{min}^{-1}$ and measurements were performed under a nitrogen rich atmosphere. The obtained traces are shown in Figure S6. A significant enthalpy relaxation is observed on the $T_{g}$ of PLLA (Figure S6a) and PLA (Figure S6c), and the respective $T_{g}$ 's shift to slightly higher values for the composite tapes. The cold crystallization of the PLLA occurs at a comparable temperature, regardless of the presence of the LCPs, whereas the melting temperature is slightly reduced due to the LCPs. The enthalpy corresponding to the cold crystallization and melting of the PLLA is reduced according to the weight fraction of LCP present. Upon cooling at a rate of $10{ }^{\circ} \mathrm{C} \mathrm{min}^{-1}$, the pure PLLA tape has a crystallization peak at $96{ }^{\circ} \mathrm{C}$, whereas crystallization does not occur in the tapes containing LCPs (Figure S6b). In the composite tapes, the $T_{g}$ 's of the dispersed LCP phase $\left(\sim 94{ }^{\circ} \mathrm{C}\right)$ and PLLA matrix phase $\left(\sim 55^{\circ} \mathrm{C}\right)$ are clearly observed. Neither cold crystallization upon heating (Figure S6c), nor crystallization upon cooling (Figures S6d) are observed in the melt drawn PLA tape or composites with the amorphous PLA as a matrix. The $T_{g}$ 's of the respective LCPs and PLA are visible in each trace, and comparable to those of the melt drawn tapes with the PLLA matrix. 
a

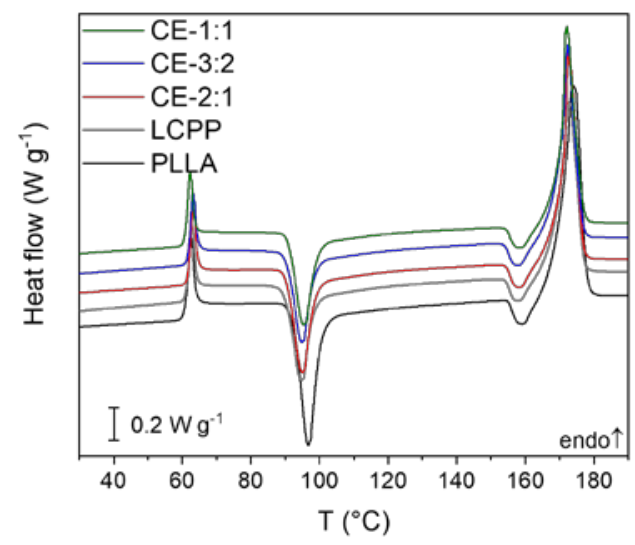

c

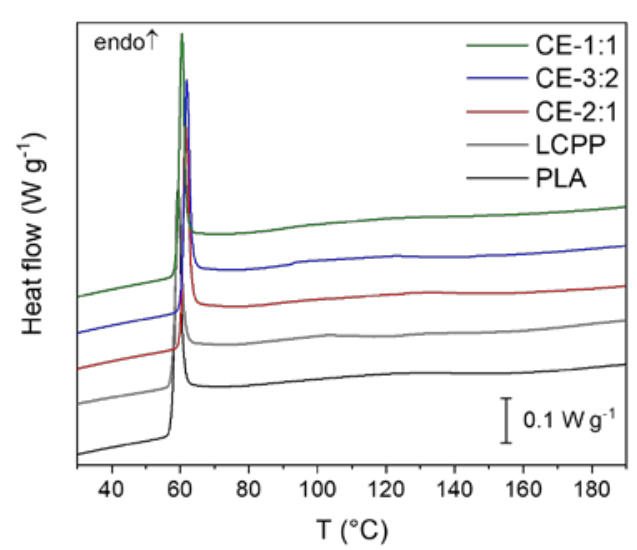

b

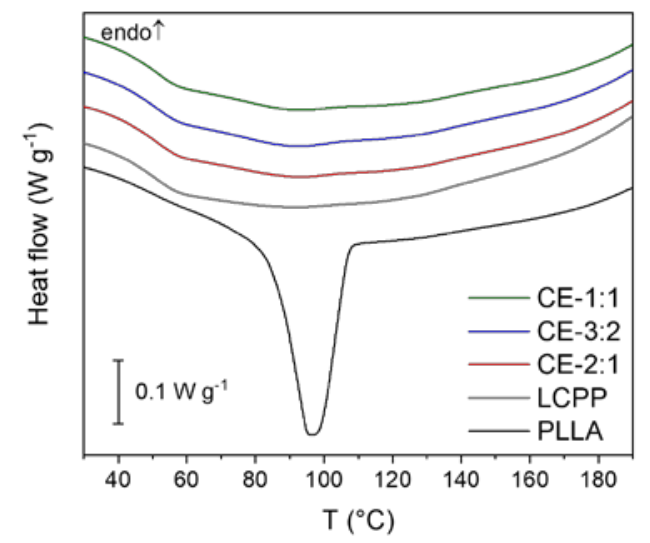

d

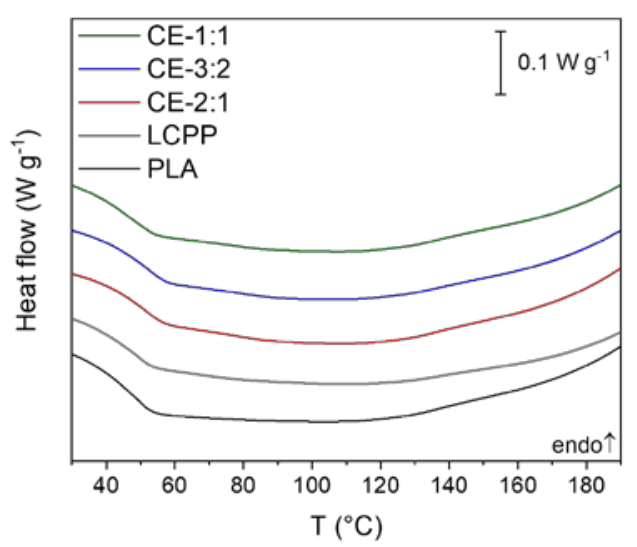

Figure S6. (a) First heating trace of melt drawn tapes containing semi-crystalline PLLA and the LCPs. Measured at $10{ }^{\circ} \mathrm{C} \mathrm{min}{ }^{-1}$. (b) First cooling trace of melt drawn tapes containing semi-crystalline PLLA and the LCPs. Measured at $10^{\circ} \mathrm{C} \mathrm{min}-1$. (c) First heating trace of melt drawn tapes containing amorphous PLA and the LCPs. Measured at $10^{\circ} \mathrm{C} \mathrm{min}^{-1}$. (d) First cooling trace of melt drawn tapes containing amorphous PLA and the LCPs. Measured at $10{ }^{\circ} \mathrm{C} \mathrm{min}^{-1}$.

\section{Diffraction patterns of melt drawn tapes with PLLA}

The WAXD diffraction patterns of the melt drawn tapes show an isotropic PLLA matrix phase (diffuse ring at slightly lower q) and an oriented LCP phase (diffuse arc at slightly higher q) prior to heating (Figure S7, Top). Upon heating, cold crystallization of the PLLA occurs. Though the PLLA appears isotropic, oriented crystals are formed and the orientation is effected by the different LCPs (Figure S7, Middle). In the tapes containing LCPs the azimuthal intensity of the PLLA crystals shows a 6 -fold symmetry, with signal corresponding to $0^{\circ}, 60^{\circ}$ and $120^{\circ}$. The reflection at $0^{\circ}$ corresponds to chains oriented according to the drawing direction and is more dominant in 
the tapes containing the LCPs with lower molecular weights (LCPP and CE-2:1). The signals at $60^{\circ}$ and $120^{\circ}$ become stronger as the LCPs molecular weight increases, due to twisted lamellae formed by residual stresses. The LCP orientation is largely maintained even as the tapes are heated to $170{ }^{\circ} \mathrm{C}$, as can be seen by the presence of diffuse scattering arcs (Figure S7, Bottom). The signal intensity of the oriented LCPs is relatively low due to the increased diffraction due to the PLLA crystallites.

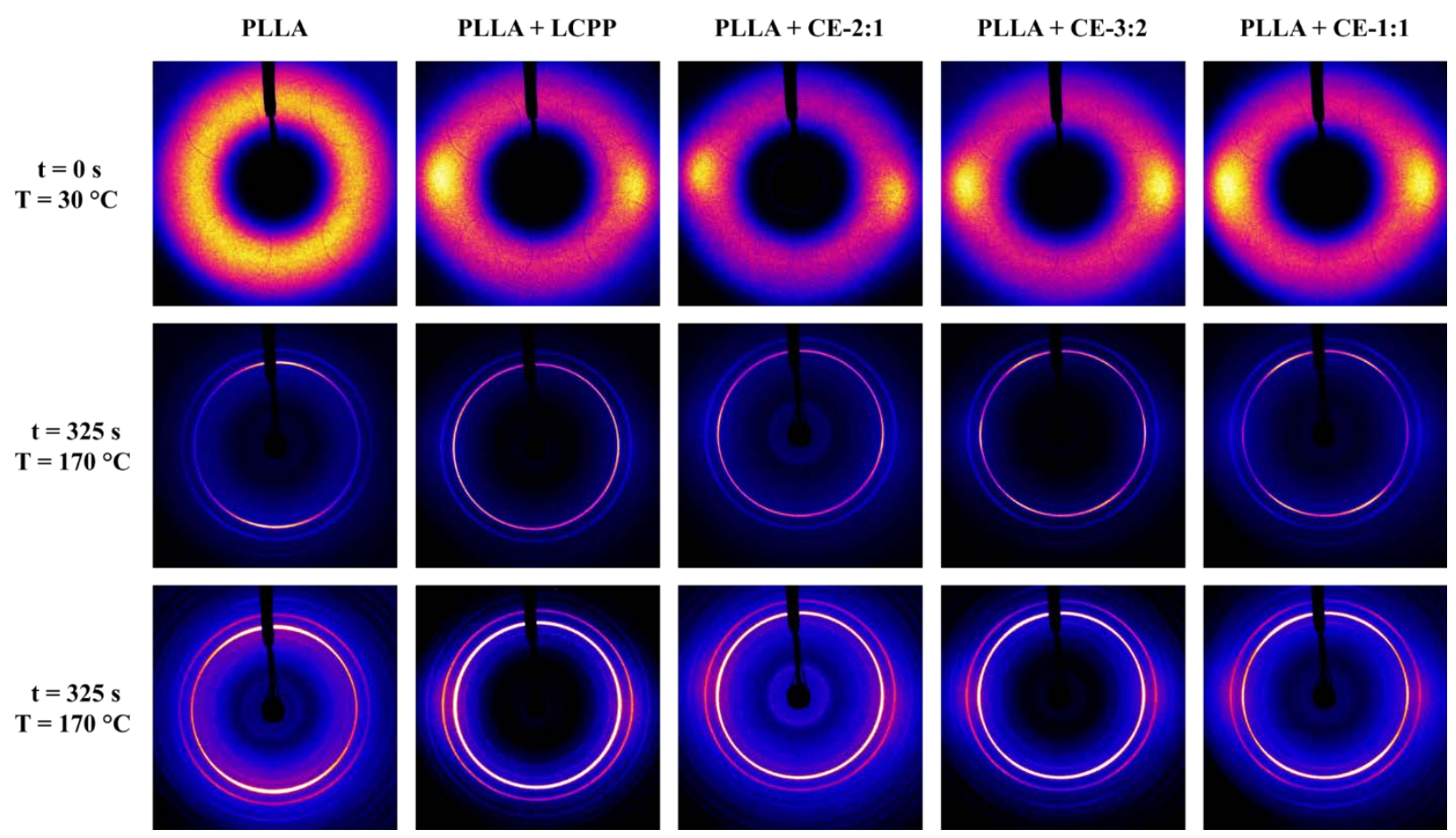

Figure S7. (Top) Diffraction patterns of the melt drawn LCP-PLLA tapes and the pure PLLA tapes, as measured prior to heating. (Middle) Diffraction pattern of the same tapes upon heating to $170{ }^{\circ} \mathrm{C}$, the intensity is set to highlight the signal corresponding to the PLLA crystals. (Bottom) Diffraction pattern of the same tapes upon heating to 170 ${ }^{\circ} \mathrm{C}$, the intensity is set show the remaining orientation of the LCP phase. 